\title{
Information retrieval, decision making process and user needs in the context of ubiquitous and collaborative computing
}

\author{
Rahat Iqbal
}

Published online: 29 October 2013

(C) Springer-Verlag Berlin Heidelberg 2013

Mark Weiser put forth a vision of ubiquitous computing, according to which people and environments are augmented with computational resources which provide information and services whenever and wherever required. Such systems or services feature multi-device architectures and complex interactions. They are often part of the environment, where they host a range of everyday activities in ways that imply new kinds of user involvement. With these sorts of systems, it is important to understand the way systems and people work in the environments which are to be augmented. This allows bringing multi-disciplinary researchers together in order to discuss different models and theories to design and evaluate ubiquitous and collaborative computing systems and services. Given these issues, we aimed to balance technological innovation with adequate consideration for the needs of users at the International Workshop on Ubiquitous and Collaborative Computing (iUBICOM) forum.

A particular focus is on how user needs can be interpreted given new interaction models and unfamiliar technologies. We discussed the challenge of understanding user needs around factors such as speed, transparency, efficiency and quality of service. Applications included wayfinding systems, smart homes, assistive technologies, personalised information retrieval and collaborative systems. The emphasis was in the consideration of human factors when designing and developing systems that can be deployed successfully.

This special issue of the International Journal of Ambient Intelligence and Humanized Computing (AIHC)

R. Iqbal $(\bowtie)$

Faculty of Engineering and Computing, Coventry University,

CV1 5FB Coventry, UK

e-mail: r.iqbal@coventry.ac.uk is on information retrieval, decision making processes and user needs in the context of ubiquitous and collaborative computing. The motivation for this special issue is to deepen our understanding of how individual user or user groups' needs can be supported by information retrieval, decision making and health monitoring systems. For example, what information about a user's needs does a system need to be aware of, and how can this be gathered? How can this process be automated? Can systems adapt to changing user needs, including changes of context and task?

The iUBICOM which was conducted in conjunction with the British Conference on Human Computer Interaction in 2011 has attracted a range of high quality submission from researchers working in the areas from information retrieval, through decision making, to contextaware service discovery and health monitoring systems. This issue consists of six high-quality papers. All submissions were considered and subjected to a rigorous review process according to AIHC standards.

The first paper, by Babak Akhgar, Esmael Salahi Parvin, Mohammad Hussein Sherkat, presents an axiomatic agent based architecture for agile decision making in strategic information systems in uncertain environments. The proposed approach is based on emotional agents and axiomatic design theory which considers critical points such as emotional decision making and flexibility in agile strategic information systems.

The second paper, by Saeedeh Maleki-Dizaji, Jawed Siddiqi, Yasaman Soltan-Zadeh, Fazilatur Rahman, addresses the problem of information overload by proposing an adaptive agent-based modelling approach that relies on evolutionary user-modelling. The approach uses both qualitative (subjective) user relevance feedback as well as quantitative (algorithmic) measures of the 
relevance of retrieved documents. It is shown that the results can be improved by applying computational intelligence techniques for modelling information needs, through interactive reinforcement learning. The approach performed better in terms of Precision and Recall as compared to the existing traditional approaches.

The third paper, by Saad Liaquat Kiani, Ashiq Anjum, Nick Antonopoulos, Michael Knappmeyer, presents a paper entitled context-aware service utilization in the clouds and energy conservation. The paper discusses the concept of a context-brokering component to support coordination and communication between mobile devices and services deployed in a cloud infrastructure. In these days, mobile devices are increasingly used for human interaction and importantly for service utilization through cloud computing. However, energy consumption still remains the main issue. The approach proposed in this paper results in reduction in energy consumption when accessing and coordinating with cloud services on a smart device.

The fourth paper, by Kashif Iqbal, Michael O. Odetayo and Anne James, presents a new approach for face detection of ubiquitous surveillance images for biometric security from an image enhancement perspective. This paper addresses the issues related to the poor visibility of the images produced by surveillance cameras by proposing an integrated image enhancement approach for face detection. The proposed approach is based on contrast enhancement and colour balancing methods. The contrast enhancement method is used to improve the contrast, while the colour balancing method helps to achieve a balanced colour. The results show a significant improvement in face detection by using the proposed approach, as compared to the traditional approaches.

The fifth paper, by Faiyaz Doctor, Rahat Iqbal, Raouf N. G. Naguib, presents a fuzzy ambient intelligent agents approach for monitoring disease progression of dementia patients. This paper discusses the development of an ambient intelligent-based system for the monitoring of dementia patients living in their own homes. Within this system groups of unobtrusive wireless sensor devices are deployed at specific locations within a patient's home and accessed via standardized interfaces provided through an open middleware platform. For each sensor group intelligent agents are used to learn fuzzy rules, which model the patient's habitual behaviours in the environment. The proposed system reports macro level behaviour changes and micro level perception drift to care providers to enable them to make better-informed assessments of the patient's cognitive abilities and changing care needs.

The sixth paper, by Farzad Amirjavid, Abdenour Bouzouane, Bruno Bouchard, discusses an approach for monitoring Alzheimer patients living in smart homes in order to facilitate in their daily activities. The approach is based on fuzzy clustering technique, and it emphasizes on applying sensors to provide primary data about realization patterns of actions, operations, plans, goals and generally any objective that the smart home resident may desire to do. Spatio temporal data related to daily activities are monitored to discover the patterns of activities carried out by Alzheimer patients in order to facilitate them. 